\title{
On the design of integral observers for unbiased output estimation in the presence of uncertainty
}

\author{
L. Bodizs ${ }^{a *}$, B. Srinivasan ${ }^{b}$ and D. Bonvin ${ }^{a \dagger}$ \\ ${ }^{a}$ Laboratoire d'Automatique, Ecole Polytechnique Fédérale de Lausanne \\ CH-1015 Lausanne, Switzerland \\ ${ }^{b}$ Department of Chemical Engineering, Ecole Polytechnique Montreal \\ Montreal H3C 3A7, Canada
}

October 5, 2010

\begin{abstract}
Integral observers are useful tools for estimating the plant states in the presence of non-vanishing disturbances resulting from plant-model mismatch and exogenous disturbances. It is well known that these observers can eliminate bias in all states, given that as many independent measurements are available as there are independent sources of disturbance. In the most general case, the dimensionality of the disturbance vector affecting the plant states corresponds to the order of the system and thus all states need to be measured. This condition, which is termed integral observability in the literature, represents a fairly restrictive situation. This study focuses on the more realistic case, where only the output variables are measured. Accordingly, the objective reduces to the unbiased estimation of the output variables. It is shown that both stability and asymptotically unbiased output estimation can be achieved if the system is observable, regardless of the dimensionality of the disturbance vector. Furthermore, a condition is provided under which, using output measurements, the errors in all states can be pushed to zero. It is also proposed to use off-line output measurements to tune the observer using a calibration-like approach. Integral observers and integral Kalman filters are evaluated via the simulation of a fourth-order linear system perturbed by unknown non-vanishing disturbances.
\end{abstract}

Keywords: Integral observer; integral Kalman filter; integral observability; plant-model mismatch; robust estimation; unbiased estimation.

\section{Introduction}

State estimation is a necessary component of process monitoring and control, as these techniques often require information that is too expensive or impossible to obtain from direct measurements. Estimation attempts to reconstruct the missing information from both the output measurements

\footnotetext{
${ }^{*}$ Current address: BASF SE, GCP/RS, L 511, D-67063 Ludwigshafen, Germany

${ }^{\dagger}$ Corresponding author. Email: dominique.bonvin@epfl.ch
} 
and prior knowledge in the form of a dynamic model [11]. For linear systems with perfect model information and gaussian measurement noise, the optimal estimator is the Kalman filter (KF) [16]. Unfortunately, the models used for state estimation are rarely accurate, and there is a lot of uncertainty in the form of plant-model mismatch and exogenous disturbances. In the presence of uncertainty, the state estimates are typically biased. To reduce this bias, the following classes of techniques have been proposed in the literature:

1. Minmax approach. The estimator is designed for the worst scenario in terms of uncertainty, and interval bounds on the estimates are provided $[1,4]$. However, this approach can be very conservative.

2. Decoupling from unknown disturbances. The observer is designed to decouple the dynamics of the estimation errors from the unknown disturbances $[19,8,9,15]$. This way, the states and the unknown disturbances can be estimated separately. However, the conditions necessary for decoupling might be difficult to meet in practice (at least as many output measurements as there are unknown sources of disturbance), and thus applicability is limited.

3. Absorption of unknown disturbances. The effect of unknown disturbances is absorbed in auxiliary states. This is done by augmenting the state vector with additional state variables. This approach has been given various names over the decades such as bias estimation [13, 17, 18], garbage collector [10], or integral observer [3, 22]. Note that, with integral observers, unbiased estimation of all states typically requires the measurement of all states [24, 28], or of at least as many independent outputs as there are independent sources of disturbance [25], which is rather impractical.

Usually, full-state estimation is considered because of the close link between estimation theory and the full-state feedback literature [7]. In some cases, however, the goal is to control only a subset of the states, for which full-state knowledge is not required. Also, in the context of process monitoring, it often suffices to estimate accurately the variables being monitored. For these cases, it may be helpful to design observers that are capable of estimating the variables of interest more accurately than could be done via full-state estimation.

This study is concerned with the asymptotically unbiased estimation of output variables. One may ask: Why estimate the output variables since they can be measured? The main reason is of course the reduction of measurement errors through the use of "model-based" filtering, which can indeed be optimal under certain circumstances. Another reason is to be able to obtain "faster" estimates when, in certain processes, the variables of interest can only be measured at low frequency and with significant delay. In these cases, inter-sample estimates are important, for which the estimation problem has to be reformulated as a multi-rate scheme [26]. In such a scheme, some or all of the measurements are available less frequently (slow time scale) than the estimates are needed (fast time scale). One way of handling two-time-scale systems is to extrapolate the slow measurements for the fast time scale by using some kind of approximations, e.g. zero-order hold or polynomial approximations, and then use a single-rate estimation technique [27, 28]. Another approach is to update the estimates only when the slow measurements become available and, in-between, use the predictions given by the process model corrected using the available fast measurements $[2,23,5]$. This multi-rate aspect is not addressed in this paper. 
There is another question related to the estimation of output variables: Is it possible to design an integral observer that asymptotically leads to unbiased estimation of all states, not simply the measured ones? This is indeed possible if (i) the mean value of the non-vanishing disturbance vector (modeled as process noise) is known, and (ii) the disturbance directions can be "reached" by the integral states.

This paper investigates integral observers capable of accurate asymptotic estimation (filtering) of the output variables, for which the classical observability of the states from the output measurements is the only required condition. In other words, no condition on the number of measurements relative to the number of disturbance sources is imposed. In addition, this study will show how the observer can be tuned using off-line output measurements and a calibration-like procedure that is well known in the field of chemometrics [21].

Both the proportional-integral observer (PI observer) and the integral Kalman filter (IKF) will be investigated. The structure of the two estimators is the same, the main difference being the way the gain matrices are computed: constant gains computed via pole placement for the observer, and time-varying gains resulting from the solution of a Riccati differential equation expressing variance propagation. The main focus of this study concerns structural properties leading to bias elimination. This structural investigation will be done for the PI observer, IKF being solely used in the simulation to show the attainable performance in the (ideal) case where the process and measurement noise covariances are known.

The paper is organized as follows. Observer structures, including P and PI observers and KF and IKF filters, are introduced in Section 2. The possibility of bias elimination in the output variables and the important property of observer stability are discussed in Section 3. Calibration-based tuning of the PI observer is proposed in Section 4. The PI observer and IKF are evaluated for various simulated scenarios in Section 5, and conclusions are drawn in Section 6.

\section{Observers for Unbiased Estimation}

\subsection{System and uncertainty description}

Consider a plant described by the following linear time-invariant (LTI) discrete-time state-space equations:

$$
\begin{aligned}
x_{k+1} & =A x_{k}+B u_{k}+w_{k}, \quad x_{0}=x_{o}, \\
y_{k} & =H x_{k}+v_{k},
\end{aligned}
$$

where $u_{k} \in \Re^{q}$ is the input vector, $x_{k} \in \Re^{n}$ the state vector with unknown initial values $x_{o}$, and $y_{k} \in \Re^{p}$ the measured output vector. The matrix $A$ denotes the state propagation matrix, $B$ the effect of the inputs on the states, and $H$ the measurement model. The vectors $w_{k}$ and $v_{k}$ represent stationary process and measurement noises, respectively.

The basic system-theoretical assumption used in this work is:

A.1 $H$ is of rank $p$ and the pair $(A, H)$ is observable. 
The process noise vector $w_{k}$ can be thought of as being generated by the noise vector $W n_{k}$ and the additive disturbance term $D d_{k}$ as follows:

$$
w_{k}=W n_{k}+D d_{k}
$$

where $W n_{k}$ represent filtered white noise and $D d_{k}$ expresses the effect of deterministic disturbances such as parametric uncertainty, unmodeled dynamics, nonlinear or time-varying terms in systems dynamics and non-zero-mean exogenous disturbances [8]. The process noise $w_{k}$ has mean $E\left(w_{k}\right)=$ $\mu_{k}$ and covariance $Q=W W^{T}$, where $E(\cdot)$ denotes the mathematical expectation operator. On the other hand, the measurement noise vector $v_{k}$ has zero mean and covariance $R$. It is furthermore assumed that the two noise sequences are uncorrelated.

This work deals with the ability to asymptotically reject estimation errors caused by the nonvanishing disturbance vector $w_{k}$. For that purpose, we will introduce an important assumption regarding the disturbance vector:

A.2 As the plant reaches steady state, the disturbance vector $w_{k}$ goes to the (non-zero) value $\mu_{\infty}$.

This assumption is well suited for the case of plant-model mismatch since, when $x_{k}$ and $u_{k}$ go to steady state, the resulting mismatch reaches a constant value. Furthermore, the chosen framework allows dealing with time-varying disturbances, as long as the disturbances vary slowly compared to the convergence speed of the observer. This point will be discussed later and is well documented in the application of integral observers to an experimental fermentation process [5].

\section{$2.2 \quad$ Observer structures}

This subsection presents various observer and filter structures and investigates their ability to produce unbiased estimates.

\subsubsection{P observer}

The first structure studied is the Luenberger proportional observer [20]:

$$
\begin{aligned}
\hat{x}_{k+1} & =A \hat{x}_{k}+B u_{k}+K_{L O}\left(y_{k}-\hat{y}_{k}\right), \quad \hat{x}_{0}=E\left(x_{o}\right), \\
\hat{y}_{k} & =H \hat{x}_{k},
\end{aligned}
$$

where the symbol $(\hat{.})$ denotes the estimate of a variable and $K_{L O} \in \Re^{n \times p}$ the gain matrix of the Luenberger observer acting on the $y$ measurements. Combining (1) and (3), the dynamics of the estimation error $e_{x, k}=x_{k}-\hat{x}_{k}$ can be written as:

$$
E\left(e_{x, k+1}\right)=\left(A-K_{L O} H\right) E\left(e_{x, k}\right)+\mu_{k}, \quad e_{x, 0}=x_{o}-E\left(x_{o}\right) .
$$

With Assumption A.2, these dynamics reach steady state asymptotically if all eigenvalues of $(A-$ $\left.K_{L O} H\right)$ are within the unit circle. This is guaranteed if the pair $(A, H)$ is observable [7]. 


\subsubsection{Kalman filter}

The problem of state estimation can also be addressed using a Kalman filter with time-varying gains. The main advantage of using a Kalman filter is its optimality, that is, it minimizes the covariance of the estimation errors. The Kalman filter gains are obtained as the solution of a Riccati equation that involves the covariances of the process and measurement noises. The Kalman filter equations read:

$$
\begin{aligned}
\hat{x}_{k+1} & =A \hat{x}_{k}+B u_{k}+K_{K F}(k)\left(y_{k}-\hat{y}_{k}\right), \quad \hat{x}_{0}=E\left(x_{o}\right), \\
\hat{y}_{k} & =H \hat{x}_{k}, \\
K_{K F}(k) & =A P(k) H^{T}\left(R+H P(k) H^{T}\right)^{-1}, \\
P(k+1) & =A P(k) A^{T}+Q-A P(k) H^{T}\left(R+H P(k) H^{T}\right)^{-1} H P(k) A^{T},
\end{aligned}
$$

where $K_{K F}(k) \in \Re^{n \times p}$ denotes the Kalman gain matrix at the time instant $k$ and $P(k) \in \Re^{n \times n}$ the estimation error covariance matrix.

Often, the steady-state solution $K_{K F}$ is used, which corresponds to a fixed-gain $\mathrm{P}$ observer, the main difference being the way the gains are computed. In a $\mathrm{P}$ observer, the gains are chosen via pole placement, with the pole locations being the key decision, while the Kalman filter computes gains that minimize the covariance of the estimation errors based on the assumed knowledge of the process and measurement noise covariances.

\subsubsection{PI observer}

As in the context of feedback control, integral action can be used to remove the bias in estimates. The structure proposed here is a linear proportional-integral observer based on the $y$ measurements:

$$
\begin{aligned}
\hat{x}_{k+1} & =A \hat{x}_{k}+B u_{k}+K^{x}\left(y_{k}-\hat{y}_{k}\right)+K^{i} \alpha_{k}, & & \hat{x}_{0}=E\left(x_{o}\right), \\
\alpha_{k+1} & =\alpha_{k}+K^{\alpha}\left(y_{k}-\hat{y}_{k}\right), & & \alpha_{0}=0 \\
\hat{y}_{k} & =H \hat{x}_{k}, & &
\end{aligned}
$$

where the integral states $\alpha \in \Re^{p}$ are built from the output errors using the gain matrix $K^{\alpha} \in \Re^{p \times p}$, while $K^{x} \in \Re^{n \times p}$ is the proportional gain matrix acting on the $y$ measurements, and $K^{i} \in \Re^{n \times p}$ the gain matrix acting on the integral states. The dynamics of the augmented $(n+p)$-dimensional estimation error $\bar{e}_{k}$ is:

$$
\begin{array}{cc}
E\left(\bar{e}_{k+1}\right)=(\bar{A}-\bar{K} \bar{H}) E\left(\bar{e}_{k}\right)+\bar{\mu}_{k}, & \\
\bar{e}_{k}=\left[\begin{array}{c}
e_{x, k} \\
\alpha_{k}
\end{array}\right], \quad \bar{A}=\left[\begin{array}{cc}
A & K^{i} \\
0_{p \times n} & I_{p}
\end{array}\right], \\
\bar{K}=\left[\begin{array}{c}
K^{x} \\
K^{\alpha}
\end{array}\right], \quad \bar{H}=\left[\begin{array}{ll}
H & 0_{p \times p}
\end{array}\right], \quad \bar{\mu}_{k}=\left[\begin{array}{c}
\mu_{k} \\
0_{p}
\end{array}\right] .
\end{array}
$$

Arbitrary pole placement for the errors dynamics requires here the observability of the pair $(\bar{A}, \bar{H})$, which will be discussed in detail in the next section. Also therein, the convergence of the error vector to zero will be analyzed. 


\subsubsection{Integral Kalman filter}

In the same manner a P observer is extended to a PI observer, a Kalman filter can be extended to an integral Kalman filter. The IKF structure is [16]:

$$
\begin{array}{rlr}
\hat{x}_{k+1} & =A \hat{x}_{k}+B u_{k}+K_{K F}^{x}(k)\left(y_{k}-\hat{y}_{k}\right)+K^{i} \alpha_{k}, & \hat{x}_{0}=E\left(x_{o}\right), \\
\alpha_{k+1} & =\alpha_{k}+K_{K F}^{\alpha}(k)\left(y_{k}-\hat{y}_{k}\right), & \alpha_{0}=0, \\
\hat{y}_{k} & =H \hat{x}_{k}, \\
K_{K F}^{x}(k) & =\left(A P(k) H^{T}+K^{i} P_{x \alpha}^{T} H^{T}\right)\left(R+H P(k) H^{T}\right)^{-1} \\
K_{K F}^{\alpha}(k) & =P_{x \alpha}^{T}(k) H^{T}\left(R+H P(k) H^{T}\right)^{-1} \\
\bar{P}(k+1) & =\bar{A} P(k) \bar{A}^{T}+\bar{Q}-\bar{A} \bar{P}(k) \bar{H}^{T}\left(R+\bar{H} P(k) \bar{H}^{T}\right)^{-1} \bar{H} \bar{P}(k) \bar{A}^{T},
\end{array}
$$

with

$$
\bar{P}=\left[\begin{array}{cc}
P & P_{x \alpha} \\
P_{x \alpha}^{T} & P_{\alpha}
\end{array}\right], \quad \bar{Q}=\left[\begin{array}{cc}
Q & 0 \\
0 & 0
\end{array}\right],
$$

where the integral states $\alpha \in \Re^{p}$ are built from the output errors using the time-varying gain matrix $K_{K F}^{\alpha}(k) \in \Re^{p \times p}$, while $K_{K F}^{x}(k) \in \Re^{n \times p}$ is the Kalman gain matrix at the time instant $k$, and $K^{i} \in \Re^{n \times p}$ the gain matrix acting on the integral states. $P_{\alpha}$ denotes the variance-covariance of the integral states and $P_{x \alpha}$ the covariance between the states $x$ and the integral states $\alpha$.

Convergence of the Riccati equation requires the observability of the pair $(\bar{A}, \bar{H})$. Also, at steady state, IKF corresponds to a PI observer and thus shares the same convergence properties. Again, the main difference between the PI observer and IKF lies in the way the filter gains are computed.

\section{Analysis of PI observer}

\subsection{Asymptotic error analysis for $\mathbf{P}$ observer}

For the $\mathrm{P}$ observer, the steady-state error (i.e. for $k \rightarrow \infty$ ) is given by:

$$
E\left(e_{x, \infty}\right)=\left(I-A+K_{L O} H\right)^{-1} \mu_{\infty} .
$$

Note that, if the system is observable, it is not possible to have $E\left(e_{x, \infty}\right)=0$ since $\left(A-K_{L O} H\right)$ has all its eigenvalues in the unit circle [7]. Hence, the bias cannot be eliminated in all states with a finite-gain $\mathrm{P}$ observer. However, the bias can be reduced with high-gain observers $[6,14]$.

On the other hand, if the filtering problem concerns only the estimation of the output variables, then it is possible to choose the observer gain $K_{L O}$ such that

$$
E\left(e_{y, \infty}\right)=E\left(H e_{x, \infty}\right)=H\left(I-A+K_{L O} H\right)^{-1} \mu_{\infty}=0
$$

for a particular $\mu_{\infty}$. Hence, with a $\mathrm{P}$ observer, the bias in the measured states can be eliminated asymptotically if the mean of the process noise is known and the observer gain $K_{L O}$ designed appropriately. However, if $\mu_{\infty}$ is unknown, or if it changes, unbiased estimation of the outputs is not possible. 


\subsection{Asymptotic error analysis for PI observer}

This section first shows that the PI observer generates unbiased estimates of the output variables. This result is presented in the next theorem.

Theorem 1. Consider the system (1) with a process noise that is asymptotically non-zero mean, i.e. $\mu_{\infty} \neq 0$, and the PI observer (6). Let the gains $K^{x}, K^{\alpha}$ and $K^{i}$ of the PI observer be chosen so that $(\bar{A}-\bar{K} \bar{H})$ is Schur stable. Then, $E\left(e_{y, \infty}\right)=0$.

Proof. The hypothesis that $(\bar{A}-\bar{K} \bar{H})$ is Schur stable implies that $(I-\bar{A}+\bar{K} \bar{H})$ is full rank. The steady-state values can be calculated from the following equation:

$$
\left[\begin{array}{cc}
I-A+K^{x} H & -K^{i} \\
K^{\alpha} H & 0
\end{array}\right]\left[\begin{array}{c}
E\left(e_{x, \infty}\right) \\
E\left(\alpha_{\infty}\right)
\end{array}\right]=\left[\begin{array}{c}
\mu_{\infty} \\
0_{p}
\end{array}\right]
$$

The fact that $(I-\bar{A}+\bar{K} \bar{H})$ is full rank implies that the last rows of (11) are independent, i.e. $K^{\alpha} H$ is full rank. This, in turn, guarantees that $K^{\alpha}$ is full rank. Hence, $K^{\alpha} H E\left(e_{x, \infty}\right)=0$ leads to the error condition $E\left(H e_{x, \infty}\right)=E\left(e_{y, \infty}\right)=0$.

The above theorem states that the integral observer is able to push the estimation errors from the measured outputs to the integral states, thereby leading to unbiased estimation of the measured outputs, i.e. $E\left(e_{y, \infty}\right)=0$. Another important feature of the PI observer is that it can generate unbiased estimates of all states provided the bias $\mu_{\infty}$ is in the span of $K^{i}$. This result is presented in the following theorem.

Theorem 2. Consider the system (1) with a process noise that is asymptotically non-zero mean, i.e. $\mu_{\infty} \neq 0$, and the PI observer (6). Let the gains $K^{x}, K^{\alpha}$ and $K^{i}$ of the PI observer be chosen so that $(\bar{A}-\bar{K} \bar{H})$ is Schur stable. If $\mu_{\infty}$ is in the span of $K^{i}$, i.e. $\mu_{\infty}=K^{i} c_{i}$ for some $c_{i} \in \Re^{p}$, or equivalently if $\left(I_{n}-K^{i}\left(K^{i}\right)^{+}\right) \mu_{\infty}=0$, then $E\left(e_{x, \infty}\right)=0$.

Proof. Since $\mu_{\infty}=K^{i} c_{i}$, (11) can be written as

$$
\left[\begin{array}{cc}
I-A+K^{x} H & -K^{i} \\
K^{\alpha} H & 0
\end{array}\right]\left[\begin{array}{c}
E\left(e_{x, \infty}\right) \\
E\left(\alpha_{\infty}\right)+c_{i}
\end{array}\right]=\left[\begin{array}{c}
0_{n} \\
0_{p}
\end{array}\right]
$$

The hypothesis that $(\bar{A}-\bar{K} \bar{H})$ is Schur stable implies that $(I-\bar{A}+\bar{K} \bar{H})$ is full rank. This in turn implies $E\left(e_{x, \infty}\right)=0$ and $E\left(\alpha_{\infty}\right)=-c_{i}$.

At this point, it is interesting to compare the performance of the $\mathrm{P}$ and PI observers for $\mu_{\infty} \neq 0$. With a $\mathrm{P}$ observer, it is impossible to remove the errors in all states; if $\mu_{\infty}$ is constant and known and the observer designed appropriately, then the output errors can be pushed to zero. In contrast, a PI observer obeying the conditions given in Theorem 2 guarantees unbiased output estimates. In addition, under certain conditions, the error in all states can be pushed to zero.

It is also interesting to note that the dimension of $\operatorname{span}$ of $K^{i}$ is $\operatorname{rank}\left(K^{i}\right)=p$, i.e. the number of outputs. Hence, once $K^{i}$ is chosen, $\mu_{\infty}$ can live in a $p$-dimensional space without having any effect 
on the estimation of any of the states. In other words, as the number of outputs is increased, there is more flexibility to accommodate the disturbances and still provide unbiased state estimation. The extreme case is $p=n$, for which all states can be estimated bias free without any restriction on $\mu_{\infty}$.

The above theorems indicate that unbiased output estimates in the general case, and unbiased state estimates as a special case, can be obtained if the observer is stable, i.e. if the pair $(\bar{A}, \bar{H})$ is observable. The next subsection will show that stability is guaranteed by the observability of the pair $(A, H)$.

\subsection{Stability of PI observer}

An important question regards whether or not the poles of the observer can be placed arbitrarily and thus the stability of the observer guaranteed a priori. The next theorem shows that there exists a choice of $K^{i}$ such that the pair $(\bar{A}, \bar{H})$ is indeed observable, thereby leading to arbitrary pole placement.

Theorem 3. Let the pair $(A, H)$ be observable. Then, there exists a $K^{i}$ such that the pair $(\bar{A}, \bar{H})$ is observable.

Proof. The observability matrix of the pair $(\bar{A}, \bar{H})$ is:

$$
\mathcal{O}=\left[\begin{array}{c}
\bar{H} \\
\bar{H} \bar{A} \\
\bar{H} \bar{A}^{2} \\
\bar{H} \bar{A}^{3} \\
\vdots \\
\bar{H} \bar{A}^{n+p-1}
\end{array}\right]=\left[\begin{array}{cc}
H & 0_{p \times p} \\
H A & H K^{i} \\
H A^{2} & H(A+I) K^{i} \\
H A^{3} & H\left(A^{2}+A+I\right) K^{i} \\
\vdots & \vdots \\
H A^{(n+p-1)} & H\left(A^{(n+p-2)}+\cdots+A+I\right) K^{i}
\end{array}\right] .
$$

The rank of the matrix $\mathcal{O} \in \Re^{(n+p) p \times(n+p)}$ must be $(n+p)$ for the pair $(\bar{A}, \bar{H})$ to be observable. The proof continues by choosing $(n+p)$ rows of this matrix such that the resulting matrix, termed $\overline{\mathcal{O}} \in \Re^{(n+p) \times(n+p)}$, is full rank. The procedure is as follows:

1. Denote by $h_{i}^{T}$ the $i^{\text {th }}$ row of the matrix $H$. 
2. Build the matrices $M_{1}, M_{2}, M_{12}, M_{3}$ and $M_{4}$ as follows:

$$
M_{1}=\left[\begin{array}{c}
h_{1}^{T} \\
h_{1}^{T} A \\
h_{1}^{T} A^{2} \\
\vdots \\
h_{1}^{T} A^{q_{1}-1} \\
h_{2}^{T} \\
h_{2}^{T} A \\
\vdots \\
h_{2}^{T} A^{q_{2}-1} \\
\vdots \\
h_{p}^{T} \\
h_{p}^{T} A \\
\vdots \\
h_{p}^{T} A^{q_{p}-1}
\end{array}\right], \quad M_{2}=\left[\begin{array}{c}
h_{1}^{T} A^{q_{1}} \\
h_{2}^{T} A^{q_{2}} \\
\vdots \\
h_{p}^{T} A^{q_{p}}
\end{array}\right], \quad M_{12}=\left[\begin{array}{c}
M_{1} \\
M_{2}
\end{array}\right],
$$

by choosing the vector of indices $q=\left\{q_{1}, q_{2}, \ldots, q_{p}\right\}, 0 \leq q_{i} \leq n-1$, such that $\sum q_{i}=n-p$, $M_{1}$ is of dimension $(n-p) \times n$ with $\operatorname{rank}\left(M_{1}\right)=n-p, M_{2}$ is of dimension $p \times n$ with $\operatorname{rank}\left(M_{2}\right)=p$, and $M_{12}$ is of dimension $n \times n$ with $\operatorname{rank}\left(M_{12}\right)=n$. Such a choice of $q$ is guaranteed by the fact that the pair $(A, H)$ is observable. Note that, by construction, the rows of the matrices $M_{3}$ and $M_{4}$ are linear combinations of the rows of $M_{1}$.

3. Select the $(n+p)$ rows of the matrix of $\overline{\mathcal{O}}$ based on the matrices $M_{1}-M_{4}$ as follows,

$$
\overline{\mathcal{O}}=\left[\begin{array}{cc}
M_{1} & M_{3} K^{i} \\
M_{2} & M_{4} K^{i} \\
M_{2} A & \left(M_{2}+M_{4}\right) K^{i}
\end{array}\right] .
$$


Let $K^{i} \in \Re^{n \times p}$ be a rank $p$-matrix chosen in the null space of $M_{1} \in \Re^{(n-p) \times n}$ of rank $(n-p)$. Note that $K^{i}$ is also in the null space of $M_{3}$ and $M_{4}$ since the latter are linear combinations of the rows of $M_{1}$. With this choice of $K^{i}$, the matrix $\overline{\mathcal{O}}$ becomes:

$$
\overline{\mathcal{O}}=\left[\begin{array}{cc}
M_{1} & 0 \\
M_{2} & 0 \\
M_{2} A & M_{2} K^{i}
\end{array}\right]=\left[\begin{array}{cc}
M_{12} & 0 \\
M_{2} A & M_{2} K^{i}
\end{array}\right] .
$$

To show that $\overline{\mathcal{O}}$ is full rank, it is shown that $\overline{\mathcal{O}} v=0$ implies $v=0$ :

$$
\begin{aligned}
\overline{\mathcal{O}} v & =\left[\begin{array}{cc}
M_{12} & 0 \\
M_{2} A & M_{2} K^{i}
\end{array}\right]\left[\begin{array}{l}
v_{1} \\
v_{2}
\end{array}\right]=0, \\
M_{12} v_{1} & =0 \quad \text { and } \quad M_{2} A v_{1}+M_{2} K^{i} v_{2}=0 .
\end{aligned}
$$

Since $\operatorname{rank}\left(M_{12}\right)=n, v_{1}=0$. Since $\operatorname{rank}\left(M_{2}\right)=\operatorname{rank}\left(K^{i}\right)=p, v_{2}=0$. Hence, $v=0$, and the matrices $\overline{\mathcal{O}}$ and $\mathcal{O}$ are of rank $(n+p)$.

Since the pair $(\bar{A}, \bar{H})$ is observable, arbitrary pole placement is possible, which leads to the stability of $(\bar{A}-\bar{K} \bar{H})$. Consequently, it is always possible to obtain unbiased output estimates as stated by Theorem 1.

\subsection{Integral observability}

The observability of the pair $(\bar{A}, \bar{H})$ is termed integral observability. In the literature [24, 28], integral observability is studied with more integrators than necessary, that is, $n$ integrators instead of $p$. With $K^{\alpha} \in \Re^{n \times p}$ and $K^{i}=I_{n} \in \Re^{n \times n}$, it follows that the pair $(\bar{A}, \bar{H})$ is observable if and only if $\operatorname{rank}(H)=n$, i.e. $p=n$. Note that, if $\operatorname{rank}(H)=n$ implies the observability of the pair $(A, H)[7]$, the converse is not true. Obviously, this rank condition is more restrictive than the observability requirement on the pair $(A, H)$ required by the PI observer. The fact that the condition $\operatorname{rank}(H)=n$ is not required here can be explained by the non-redundant structure of the PI observer - only as many integrators as the number of measurements are used, whereas the integral observer proposed in the literature uses $n$ integrators even when fewer variables are measured.

\section{Calibration-based Tuning}

The gain matrix $K^{i}$ acting on the integral states is chosen according to the procedure described in Section 3.3 to guarantee observability of the pair $(\bar{A}, \bar{H})$. The observer gains $K^{x}$ and $K^{\alpha}$ are typically chosen via pole placement. The Kalman gains $K_{K F}^{x}(k)$ and $K_{K F}^{\alpha}(k)$ are computed recursively according to (8) based on the assumed knowledge of $Q$ and $R$, which are typically chosen by trial and error.

In contrast, it is proposed here to tune the observers and filters via calibration using measured data. The tuning step is carried out off-line based on measurements of the outputs $y$. This approach is analogous to calibration, which has been used extensively in the field of chemometrics [21]. The 
calibration approach consists of two steps: the calibration step fixes the model, while the prediction step uses it for prediction. Similarly, the estimation procedure here also comprises two steps: (i) the tuning step, where an optimization problem is solved to compute the observer gains and fix the estimator, and (ii) the estimation step, where the estimator obtained in Step (i) is used to compute the state estimates for a different set of data.

The calibration procedure is illustrated next for the PI observer. Since $K^{i}$ is determined separately to ensure the observability of the pair $(\bar{A}, \bar{H})$, the remaining degrees of freedom are the elements of $K^{x}$ and $K^{\alpha}$. With the objective to tune the observer for the estimation of the output variables, the calibration problem can be formulated as the minimization of the mean-square estimation error of these variables:

$$
\begin{array}{rlr}
\min _{K^{x}, K^{\alpha}} & J=\sum_{k=0}^{N-1} E\left(\left(y_{k}-\hat{y}_{k}\right)^{T} S\left(y_{k}-\hat{y}_{k}\right)\right) & \\
\text { s.t. } & \hat{x}_{k+1}=A \hat{x}_{k}+B u_{k}+K^{x}\left(y_{k}-\hat{y}_{k}\right)+K^{i} \alpha_{k}, & \\
& \alpha_{k+1}=\alpha_{k}+K^{\alpha}\left(y_{k}-\hat{y}_{k}\right), & \alpha_{0}=0, \\
& \hat{y}_{k}=H \hat{x}_{k}, &
\end{array}
$$

where the matrix $S$ is introduced to appropriately weigh the various output variables. This is an off-line procedure that requires all $N$ measurements to be available simultaneously.

Note that the objective function in (13) is the mean-square error (a scalar) and not the covariance matrix that is typically used in estimation problems [16]. The reason for choosing a scalar objective function rather than a matrix is that, except for the special case of linear systems with white noise, there exists no unique estimator gain that minimizes every element of the matrix. Hence, a weighted sum of the various elements of the matrix is necessary to determine the solution. The mean-square estimation error $E\left(\left(y_{k}-\hat{y}_{k}\right)^{T} S\left(y_{k}-\hat{y}_{k}\right)\right)$, which is the trace of the matrix $E\left(\left(y_{k}-\hat{y}_{k}\right) S\left(y_{k}-\hat{y}_{k}\right)^{T}\right)$, represents one such possible weighting. The mean-square error contains two terms, the square of the bias and the variance, both weighted equally [12]. Since integral observers eliminate bias, the real issue is variance minimization.

The optimization problem (13) guarantees a stable observer if data with infinite length and infinitely many realizations are used. Since this is not the case in practice, an additional stability constraint may be necessary. Typically, the stability constraint $\left|\lambda_{i}\right|<1, \forall i=1, \ldots, n+p$, is enforced, where $\lambda_{i}$ represents the $i^{\text {th }}$ pole of the closed-loop system.

\section{Illustrative Example}

This section illustrates the performance of the observers and filters discussed above. We will consider three simulation scenarios involving constant, piecewise-constant and time-varying disturbances, respectively. 


\subsection{Problem formulation}

\subsubsection{System equations}

Consider the $4^{\text {th }}$-order LTI discrete-time model given by (1) and (2) with:

$$
\begin{aligned}
A & =\left[\begin{array}{cccc}
0.91 & 0 & 0.11 & 0 \\
0 & 0.66 & 0.13 & -0.06 \\
0 & -0.06 & 0.75 & 0.02 \\
0.10 & 0.05 & 0 & 0.80
\end{array}\right], \quad B=\left[\begin{array}{c}
-0.05 \\
0.05 \\
0.1 \\
-0.1
\end{array}\right], \quad H=\left[\begin{array}{cccc}
0 & 1 & 0 & 0 \\
0 & 0 & 1 & 0
\end{array}\right], \\
w_{k} & =n_{k}+d_{k}, \quad n_{k} \rightarrow N(0, Q), \quad v_{k} \rightarrow N(0, R), \\
Q & =\left[\begin{array}{llll}
2 & 0 & 0 & 0 \\
0 & 3 & 0 & 0 \\
0 & 0 & 5 & 0 \\
0 & 0 & 0 & 3
\end{array}\right], \quad R=\left[\begin{array}{ll}
3 & 0 \\
0 & 5
\end{array}\right],
\end{aligned}
$$

for $k=0,1, \ldots, 200$. This system has two output variables, the states $x_{2}$ and $x_{3}$, which need to be estimated as accurately as possible. It can be verified that the pair $(A, H)$ is observable.

\subsubsection{Simulation scenarios}

The simulation scenarios presented in Table 1 are considered, with the following numerical values:

$$
\begin{aligned}
& d_{\text {constant }, k}=\left[\begin{array}{llll}
3 & 6 & 4.5 & 0.6
\end{array}\right]^{T} \quad \text { for } k \in[0,200] \text {, } \\
& d_{\text {piecewise }, k}=\left\{\begin{array}{llll}
=d_{\text {constant }, k} & \text { for } k \in[0,99], \\
=\left[\begin{array}{llll}
3.9 & -6 & -6 & -2.4
\end{array}\right]^{T} & \text { for } k \in[100,200],
\end{array}\right. \\
& d_{\text {varying }, k}=\Delta A x_{k} \\
& \Delta A=\left[\begin{array}{cccc}
-0.015 & 0.045 & -0.015 & 0.015 \\
0.03 & 0.21 & -0.045 & 0.03 \\
-0.015 & -0.03 & 0.15 & -0.075 \\
0.0075 & 0 & -0.075 & 0.15
\end{array}\right] \\
& x_{\text {tune }, o}=\left[\begin{array}{llll}
20 & -30 & 50 & -30
\end{array}\right]^{T}, \quad x_{\text {estimate }, o}=-x_{\text {tune }, o}, \\
& u_{\text {tune }, k}=\frac{0.5(0.1 k)^{2.2}+20 \sin (0.05 k)-20}{e^{0.005 k}}-100, \\
& u_{\text {estimate }, k}=-0.005 k^{2}-16 \sin (0.1 k)+200 \text {. }
\end{aligned}
$$

The initial conditions and input sequences are different for the tuning and estimation steps. 
Table 1: Simulation runs with indication of the nature of the process noise (disturbances), the initial conditions and the input sequence.

\begin{tabular}{|c|c|c|c|}
\hline Simulation & $\mathbf{1}$ & $\mathbf{2}$ & $\mathbf{3}$ \\
\hline Disturbances & constant & piecewise-constant & time-varying \\
\hline \hline$w_{k}$ & $n_{k}+d_{\text {constant }, k}$ & $n_{k}+d_{\text {piecewise }, k}$ & $n_{k}+d_{\text {varying }, k}$ \\
\hline$\hat{x}_{0}$ & $x_{\text {tune }, o}$ & $x_{\text {estimate }, o}$ & $x_{\text {estimate }, o}$ \\
\hline$u_{k}$ & $u_{\text {tune }, k}$ & $u_{\text {estimate }, k}$ & $u_{\text {estimate }, k}$ \\
\hline
\end{tabular}

\subsubsection{Performance measures}

To compare the performance of the various estimation methods, Monte-Carlo simulations are carried out. 10 realizations ${ }^{1}$ that use different noise sequences $n_{k}$ and $v_{k}$ are considered. The following quantities are computed from the estimation error $e_{x, k}=x_{k}-\hat{x}_{k}$ at the $k^{t h}$ sampling instant:

- $m_{e_{k}} \cong E\left(e_{x, k}\right)$ - the $n$-dimensional vector of error means. For each state and at the time instant $k$, it represents the mean of the estimation error over various realizations.

- $\mathcal{V}_{e_{k}} \cong \operatorname{diag}\left(E\left(\left(e_{x, k}-m_{e_{k}}\right)\left(e_{x, k}-m_{e_{k}}\right)^{T}\right)\right)$ - the $n$-dimensional vector of error variances. These variations stems from the process and measurement noises considered in the simulation.

- $\Pi_{e_{k}} \cong \operatorname{diag}\left(E\left(e_{x, k} e_{x, k}^{T}\right)\right)$ - the $n$-dimensional vector of mean-square errors (MSE). These errors represent the contribution of both the error means and the error variances.

- $\Sigma_{\Pi_{e_{k}}}=\sum_{i=1}^{n} \Pi_{e_{i, k}}$ - the scalar total MSE. It represents the sum of the MSE over the $n$ states.

Additionally, in order to have a global indication of the estimator performance over the whole time interval considered, the above quantities can be summed up over the index $k$ to give:

$$
m_{e}=\sum_{k}\left|m_{e_{k}}\right|, \quad \mathcal{V}_{e}=\sum_{k} \mathcal{V}_{e_{k}}, \quad \Pi_{e}=\sum_{k} \Pi_{e_{k}}, \quad \Sigma_{\Pi_{e}}=\sum_{k} \Sigma_{\Pi_{e_{k}}} .
$$

\subsection{Comparison of various estimation schemes}

The performance of PI observers tuned using the calibration approach will be compared to that of IKF tuned using Kalman recursion (5) based on the knowledge of the true process and measurement noise covariances. Note that this very favorable situation for IKF can only be implemented in a simulation context, for which $Q$ and $R$ are perfectly known.

\footnotetext{
${ }^{1}$ Note that such a small number of realizations does not provide accurate statistical properties. However, a sufficient approximation of these properties can be obtained with minimal computational effort.
} 
Open-loop model prediction and Kalman filtering without integral action will also be shown, the goal being to illustrate that neither open-loop model prediction nor a perfectly tuned proportional observer - the $\mathrm{KF}$ - is appropriate in the presence of non-vanishing disturbances.

\subsubsection{Constant disturbances - Simulation 1}

We consider succesively open-loop model prediction, Kalman filtering, PI observer and IKF.

1. Open-loop model prediction is obtained by integration of the system given in Section 5.2.1 for the conditions given in Table 1.

2. The KF has several tuning parameters:

- $P(0)$, the initial value of the covariance matrix, is chosen to be a diagonal matrix, with large values on the diagonal, $P(0)=100 I_{n}$;

- $Q$ and $R$ are assumed to be known in this simulation study.

The closed-loop poles at steady state, i.e. the eigenvalues of $\left(A-K_{K F} H\right)$, are:

$$
\lambda_{K F}=\left[\begin{array}{lll}
0.7163 \pm 0.0951 i & 0.7635 & 0.9238
\end{array}\right]^{T} .
$$

3. The PI observer is tuned by first choosing the gain $K^{i}$ as:

$$
K^{i}=0.1\left[\begin{array}{llll}
0 & 1 & 0 & 0 \\
0 & 0 & 1 & 0
\end{array}\right]^{T},
$$

which leads to an observable pair $(\bar{A}, \bar{H})$.

The gains $K^{x}$ and $K^{\alpha}$ are tuned using calibration by numerically solving (13). The resulting closed-loop poles are:

$$
\lambda_{P I}=\left[\begin{array}{lllll}
0.3379 & 0.4745 & 0.8115 & 0.8980 \pm 0.0037 i & 0.9034
\end{array}\right]^{T} .
$$

4. The IKF is tuned as follows:

- $\bar{P}(0)$, the initial value of the covariance matrix, is chosen to be a diagonal matrix, with large values on the diagonal, $\bar{P}(0)=100 I_{n+p}$;

- $\bar{Q}$ is chosen as in (8), where $Q$ is considered to be known;

- $R$ is also known.

The resulting closed-loop poles at steady state are:

$$
\lambda_{I K F}=\left[\begin{array}{lllll}
0.6959 \pm 0.0944 i & 0.7646 & 0.9224 & 0.9946 & 0.9950
\end{array}\right]^{T} .
$$

Figure 1 presents the performance of open-loop model prediction and Kalman filtering. Without integral action, it is not surprising that the effect of the constant disturbances cannot be compensated.

Figure 2 and Table 2 compare the performance of the PI observer and IKF. The two integral estimators perform similarly well for the output variables $x_{2}$ and $x_{3}$, and much better than KF. Since the PI observer is tuned for the very same data and IKF has perfect covariance information, it is not surprising that they both perform well for $x_{2}$ and $x_{3}$. The next subsection will consider the more realistic scenario where the PI observer is not tuned with the data used for estimation. 

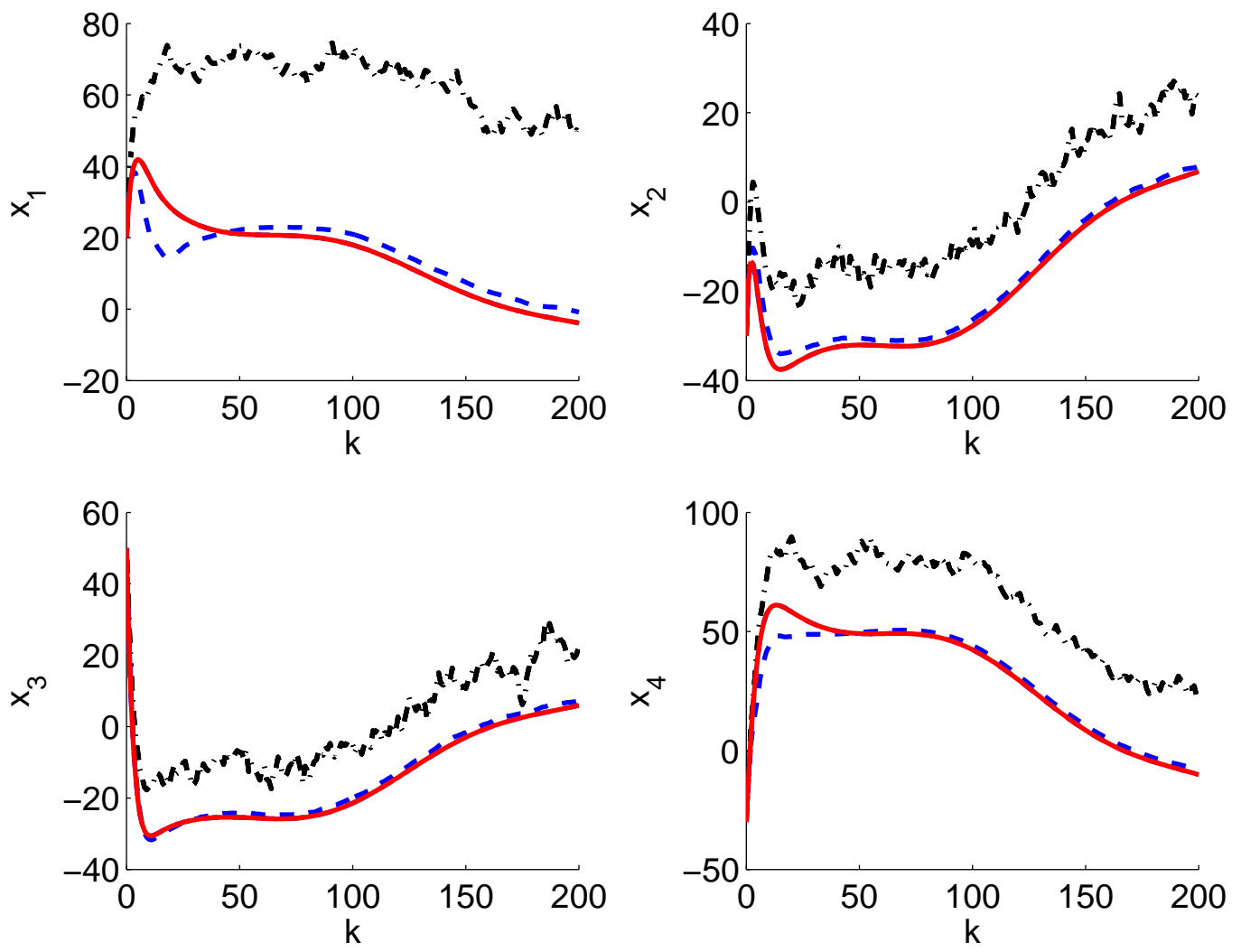

Figure 1: Open-loop model prediction and Kalman filtering for one realization in Simulation 1 (constant disturbances). Plant - (black) dash-dotted line; open-loop model prediction - (red) solid line; $\mathrm{KF}-$ (blue) dashed line.

Table 2: Performance of the PI observer and IKF in Simulation 1 (constant disturbances; PI observer tuned on the same data) for $k \in[60,200]$ and 10 realizations.

\begin{tabular}{|c|c|c|}
\hline & PI observer & IKF \\
\hline$m_{e}$ & {$\left[\begin{array}{c}4.96 \times 10^{3} \\
7.16 \times 10^{1} \\
9.18 \times 10^{1} \\
2.90 \cdot 10^{3}\end{array}\right]$} & {$\left[\begin{array}{c}4.92 \times 10^{3} \\
8.09 \times 10^{1} \\
1.41 \times 10^{2} \\
2.86 \times 10^{3}\end{array}\right]$} \\
\hline \multirow{2}{*}{$\mathcal{V}_{e}$} & {$\left[\begin{array}{c}8.91 \times 10^{3} \\
6.28 \times 10^{2} \\
9.62 \times 10^{2} \\
3.07 \times 10^{3}\end{array}\right]$} & {$\left[\begin{array}{c}2.55 \times 10^{3} \\
8.99 \times 10^{2} \\
1.40 \times 10^{3} \\
1.82 \times 10^{3}\end{array}\right]$} \\
\hline$\Pi_{e}$ & {$\left[\begin{array}{c}1.83 \times 10^{5} \\
6.80 \times 10^{2} \\
1.06 \times 10^{3} \\
6.26 \times 10^{4}\end{array}\right]$} & {$\left[\begin{array}{c}1.73 \times 10^{5} \\
9.70 \times 10^{2} \\
1.59 \times 10^{3} \\
5.97 \times 10^{4}\end{array}\right]$} \\
\hline$\Sigma_{\Pi_{e}}$ & $2.47 \times 10^{5}$ & $2.35 \times 10^{5}$ \\
\hline
\end{tabular}



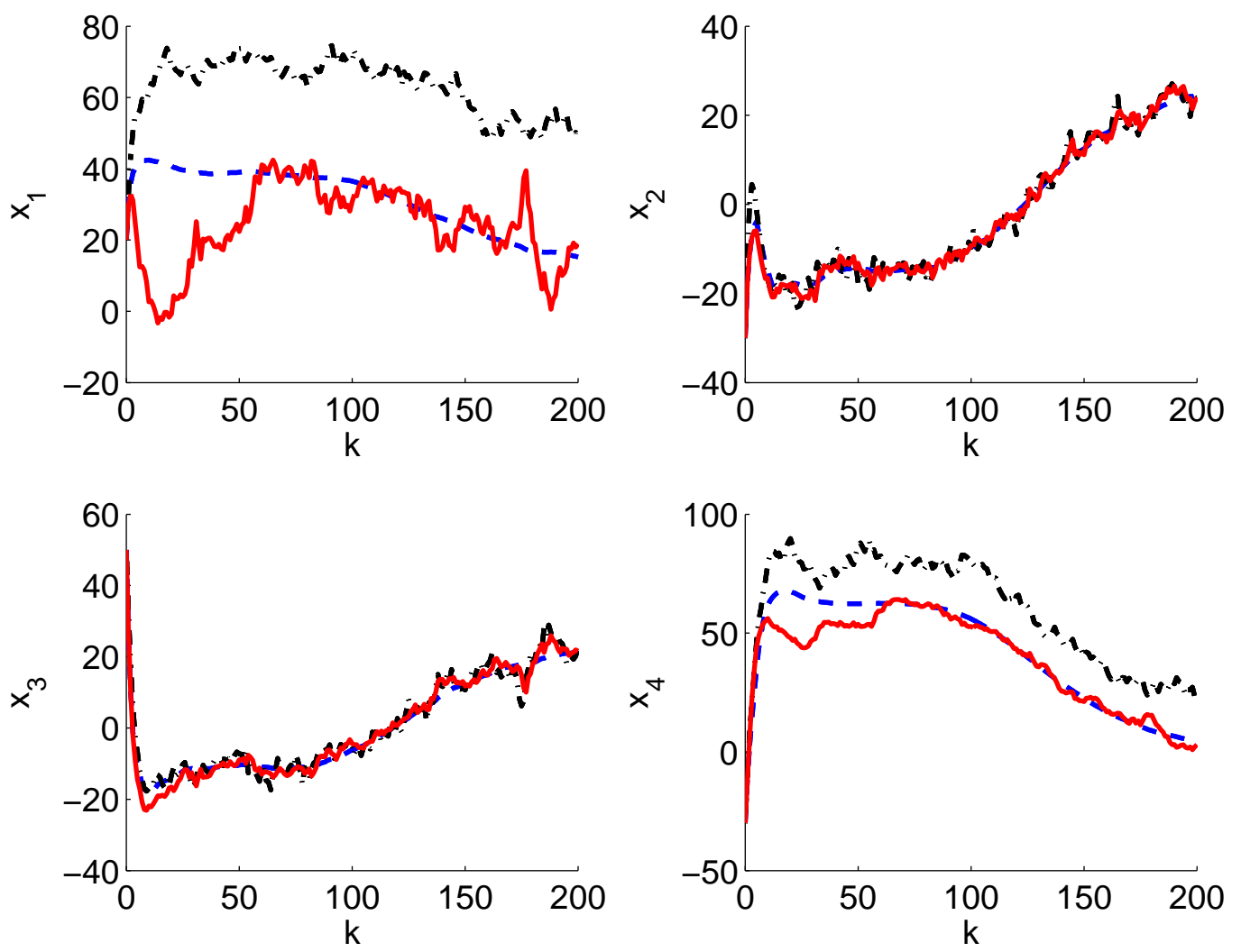

Figure 2: Performance of the PI observer and IKF for one realization in Simulation 1 (constant disturbances; PI observer tuned on the same data). Plant - (black) dash-dotted line; PI observer - (red) solid line; IKF - (blue) dashed line. 

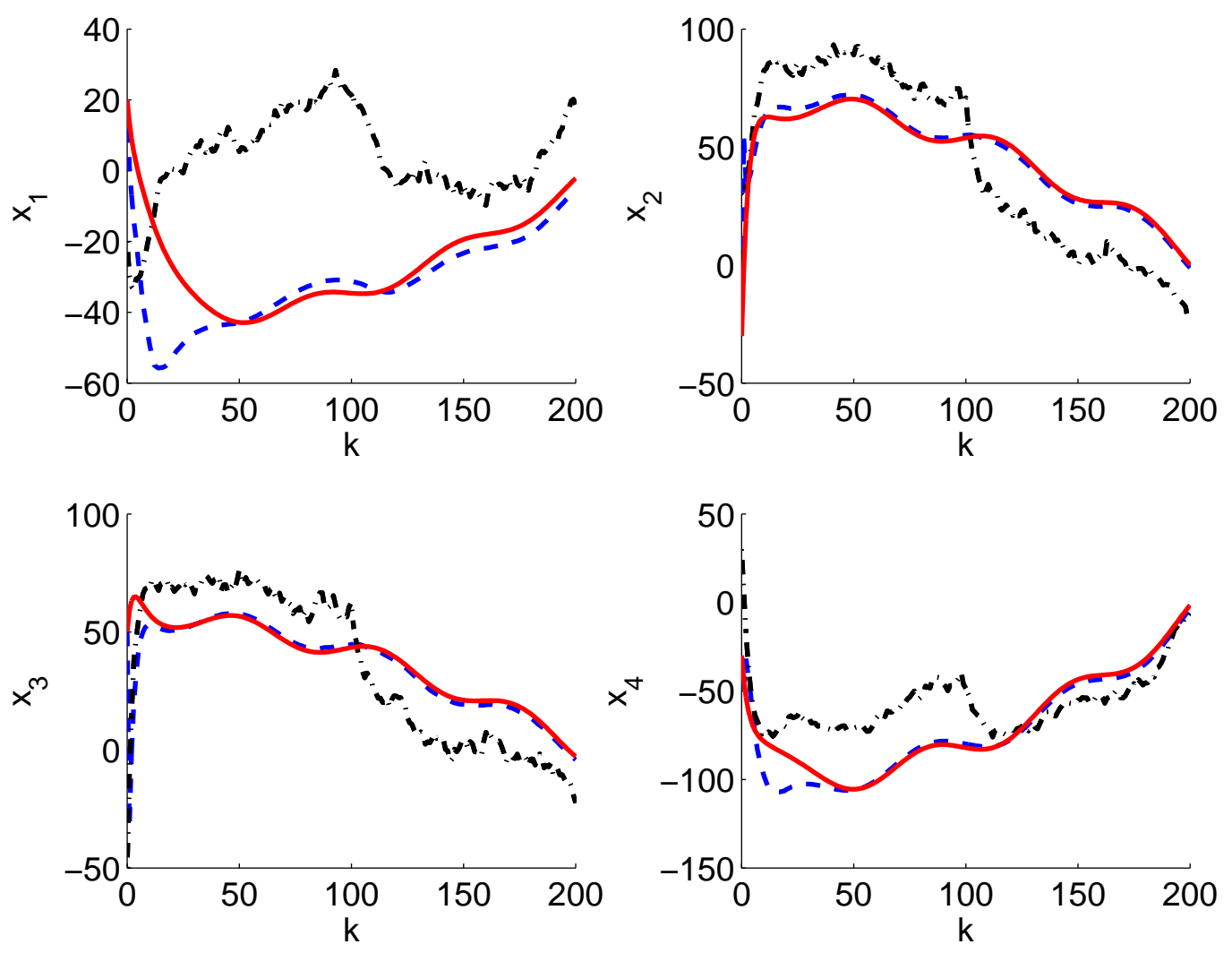

Figure 3: Open-loop model prediction and Kalman filtering for one realization in Simulation 2 (piecewise-constant disturbances). Plant - (black) dash-dotted line; open-loop model prediction (red) solid line; KF - (blue) dashed line.

\subsubsection{Piecewise-constant disturbances - Simulation 2}

Figure 3 presents the performance of open-loop model prediction and Kalman filtering. As expected, the performance is poor without integral action.

Figure 4 and Table 3 compare the performance of the PI observer and IKF in the presence of a previously unknown piecewise-constant disturbances. It is seen that the PI observer outperforms IKF regarding the estimation of the output variables $x_{2}$ and $x_{3}$. This is due to the fact that IKF reacts slowly to the disturbance change that occurs at $k=100$. Indeed, once the IKF gains have converged to their steady-state values, the filter looses its capacity to 'adapt' to the change in disturbances.

In order to improve the performance of IKF, the covariance matrix can be re-initialized at $k=100$, i.e. $\bar{P}(100)=\bar{P}(0)$ and $\alpha_{100}=\alpha_{0}$. As seen in Figure 5 and Table 3 , the re-initialized IKF performs well, even slightly better than the PI observer. However, this comparison is unfair since IKF has access to additional information compared to the PI observer, namely knowledge of the instant at which the disturbances change. In fact, if the same information is available to both observers, the PI observer outperforms IKF (not shown here). This confirms the conjecture that calibrating the 

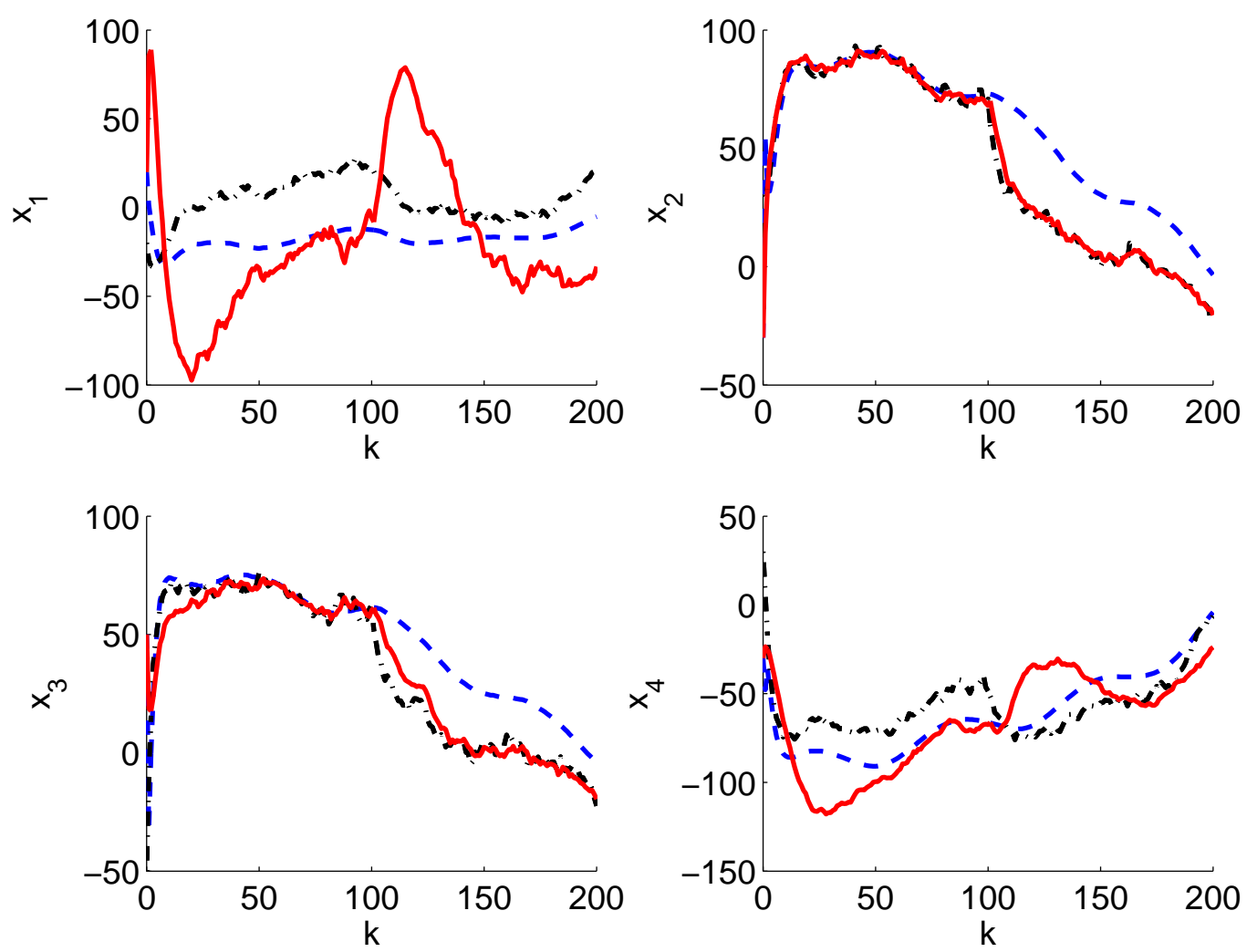

Figure 4: Performance of the PI observer and IKF for one realization in Simulation 2 (piecewiseconstant disturbances; PI observer tuned on a different set of data). Plant - (black) dash-dotted line; PI observer - (red) solid line; IKF - (blue) dashed line.

gains is at least as effective as covariance propagation based on perfect knowledge of $Q$ and $R$.

\subsubsection{Time-varying disturbances caused by plant-model mismatch - Simulation 3}

Figure 6 presents the performance of open-loop model prediction and Kalman filtering. Once again, the performance is poor for both of them.

Figure 7 and Table 4 compare the performance of the PI observer and IKF in the presence of plantmodel mismatch. As in Simulation 2, the PI observer outperforms IKF regarding the estimation of the output variables $x_{2}$ and $x_{3}$. This is due to the fact that IKF reacts slowly to the disturbance change. Looking at the closed-loop poles of the PI observer and IKF, it is seen that the absolute values are smaller for the PI observer. This implies that the dynamics of the PI observer are faster than those of IKF. For this particular simulation with plant-model mismatch, since the dynamics of the PI observer are faster than those of the time-varying disturbance, the PI observer can 'catchup'. In contrast, the dynamics of IKF, after convergence of the covariance propagation, are rather slow. Hence, repeated IKF re-initialization would be necessary to keep it alive, which however is not very practical! 

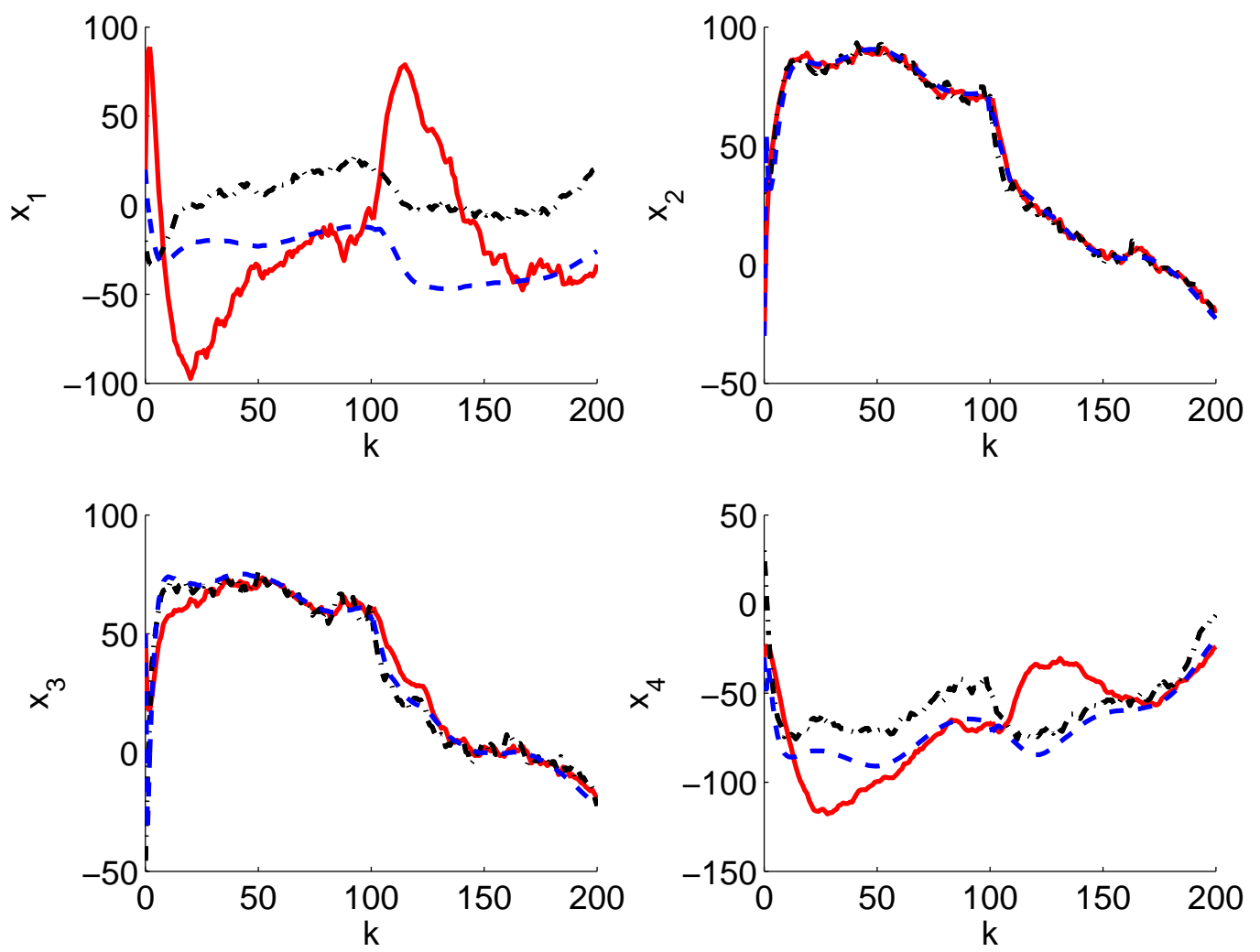

Figure 5: Performance of the PI observer and re-initialized IKF for one realization in Simulation 2 (piecewise-constant disturbances; PI observer tuned on a different set of data). Plant - (black) dash-dotted line; PI observer - (red) solid line; IKF - (blue) dashed line.

Table 3: Performance of the PI observer, IKF and re-initialized IKF in Simulation 2 (piecewiseconstant disturbances; PI observer tuned on a different set of data) for $k \in[60,200]$ and 10 realizations.

\begin{tabular}{|c|c|c|c|}
\hline & PI observer & IKF & $\mathrm{IKF}_{r e-i n i t}$. \\
\hline \multirow{4}{*}{$m_{e}$} & $5.04 \times 10^{3}$ & $3.40 \times 10^{3}$ & $5.42 \times 10^{3}$ \\
\hline & $2.08 \times 10^{2}$ & $2.71 \times 10^{3}$ & $1.74 \times 10^{2}$ \\
\hline & $4.06 \times 10^{2}$ & $2.29 \times 10^{3}$ & $1.94 \times 10^{2}$ \\
\hline & $2.39 \cdot 10^{3}$ & $1.58 \times 10^{3}$ & $1.63 \cdot 10^{3}$ \\
\hline \multirow{4}{*}{$\mathcal{V}_{e}$} & $9.03 \times 10^{3}$ & $1.58 \times 10^{3}$ & $1.41 \times 10^{3}$ \\
\hline & $6.32 \times 10^{2}$ & $8.58 \times 10^{2}$ & $8.89 \times 10^{2}$ \\
\hline & $1.14 \times 10^{3}$ & $1.74 \times 10^{3}$ & $1.75 \times 10^{3}$ \\
\hline & $3.03 \cdot 10^{3}$ & $1.63 \cdot 10^{3}$ & $1.62 \times 10^{3}$ \\
\hline \multirow{4}{*}{$\Pi_{e}$} & $2.15 \times 10^{5}$ & $8.96 \times 10^{4}$ & $2.11 \times 10^{5}$ \\
\hline & $2.02 \times 10^{3}$ & $7.66 \times 10^{4}$ & $1.52 \times 10^{3}$ \\
\hline & $4.71 \times 10^{3}$ & $5.42 \times 10^{4}$ & $2.24 \times 10^{3}$ \\
\hline & $5.88 \times 10^{4}$ & $2.33 \times 10^{4}$ & $2.37 \times 10^{4}$ \\
\hline$\Sigma_{\Pi_{e}}$ & $2.80 \times 10^{5}$ & $2.44 \times 10^{5}$ & $2.39 \times 10^{5}$ \\
\hline
\end{tabular}



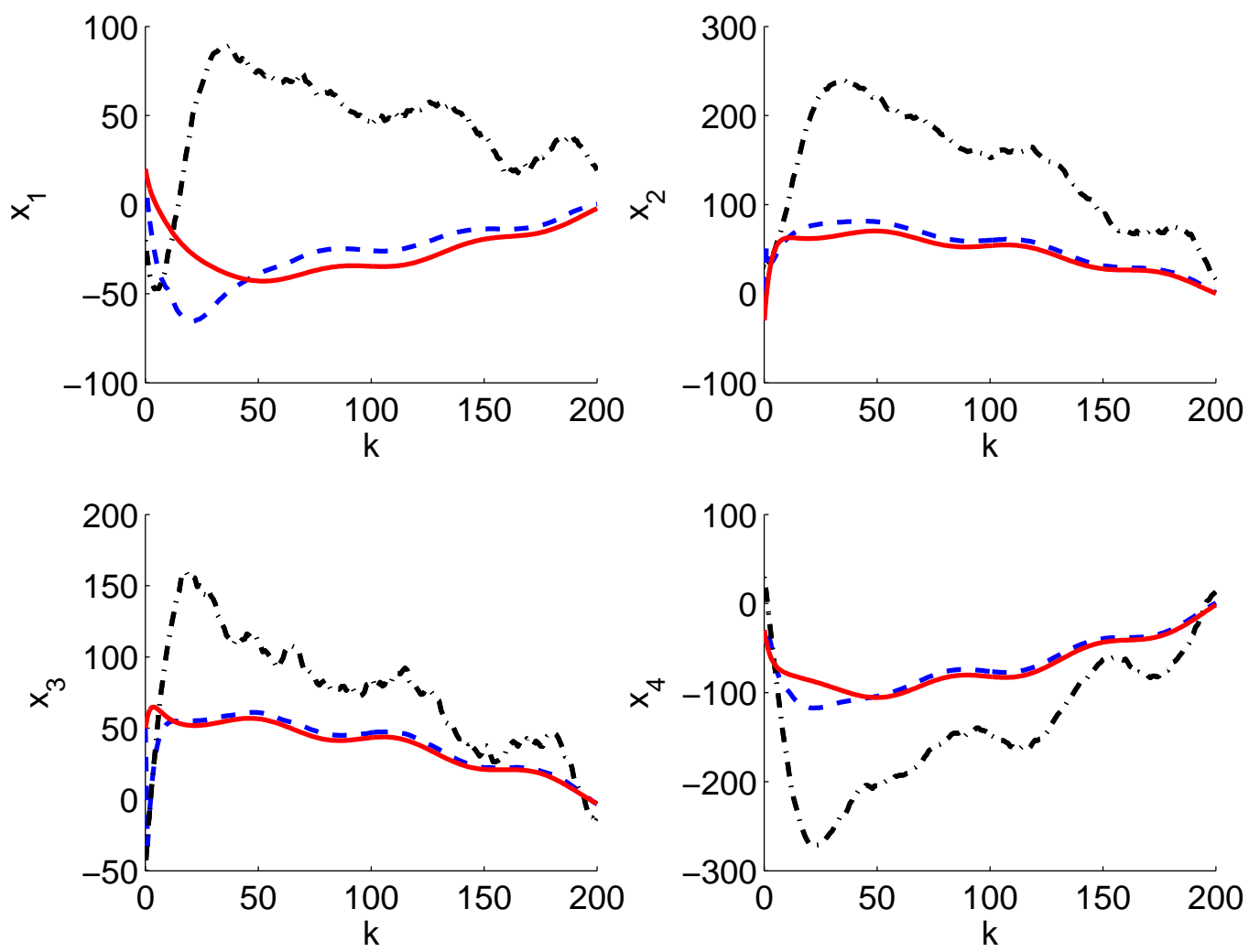

Figure 6: Open-loop model prediction and Kalman filtering for one realization in Simulation 3 (time-varying disturbances). Plant - (black) dash-dotted line; open-loop model prediction - (red) solid line; KF - (blue) dashed line. 

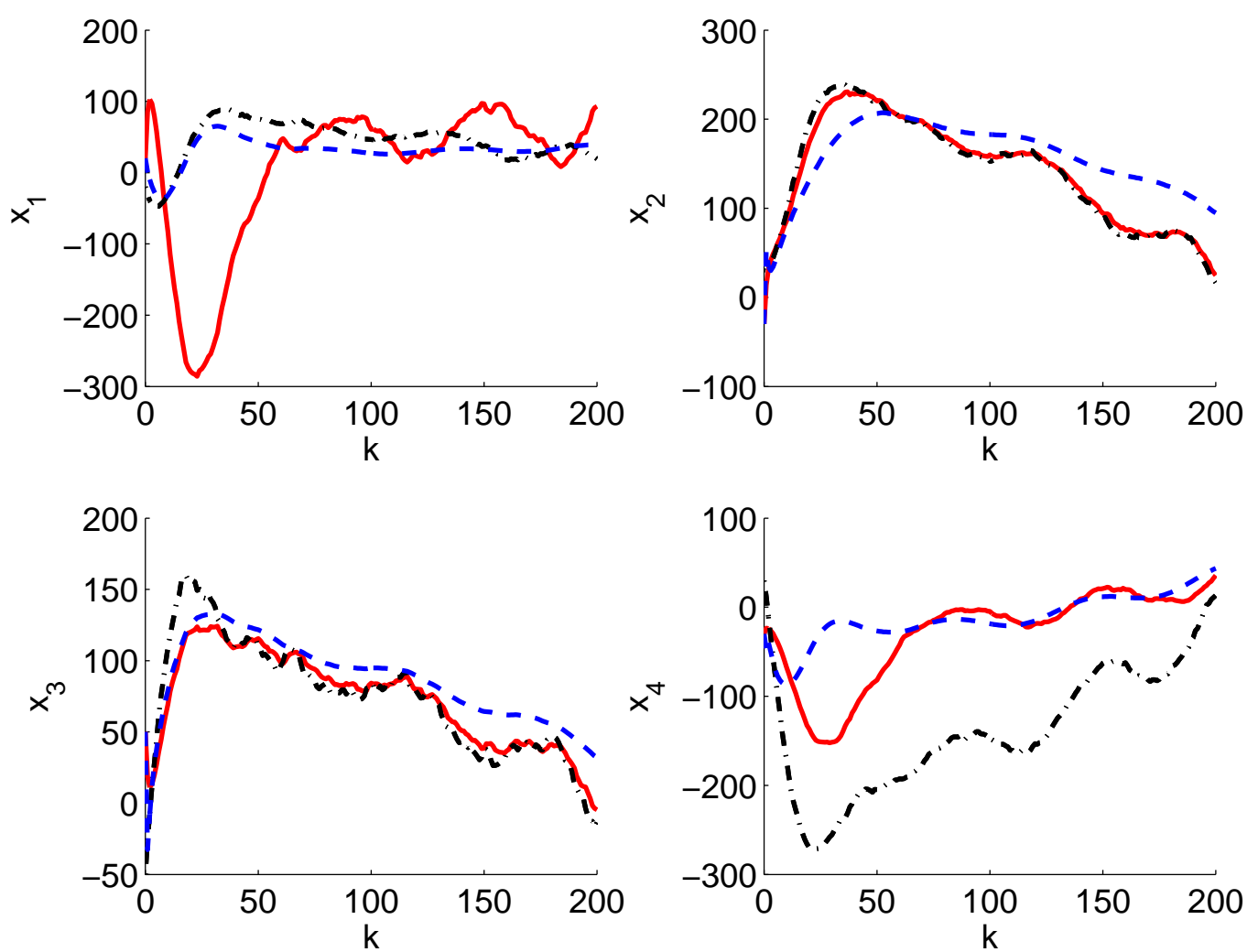

Figure 7: Performance of the PI observer and IKF for one realization in Simulation 3 (time-varying disturbances; PI observer tuned on a different set of data). Plant - (black) dash-dotted line; PI observer - (red) solid line; IKF - (blue) dashed line.

Once again, the PI observer outperforms IKF, given the same amount of information. Note that there is no theoretical guarantee that the PI observer would cope with any time-varying disturbances so well. Only asymptotic convergence for constant disturbances can be guaranteed. But, for some particular cases, the dynamics of the PI can be tuned so as to cope well with time-varying disturbances as well.

\subsubsection{Unbiased estimation based on the knowledge of $\mu_{\infty}$ - Simulation 1}

According to Theorem 2, the span of $K^{i}$ relative to the disturbance direction $\mu_{\infty}$ determines whether an integral observer can eliminate the bias in all states. If $K^{i}$ is chosen solely to produce an observable pair $(\bar{A}, \bar{H})$, unbiased estimation can only be guaranteed for the outputs. If, in addition to fulfilling this observability condition, $K^{i}$ is forced to span the disturbance space, then unbiased estimation of all states can be achieved.

In Section 5.2.1, $K^{i}$ was chosen to guarantee observability and thus lead to unbiased output estimates. In this section, we assume that $\mu_{\infty}$ is known, and the the first row of $K^{i}$ is set equal to $\mu_{\infty}$, 
Table 4: Performance of the PI observer and IKF in Simulation 3 (time-varying disturbances; PI observer tuned on a different set of data) for $k \in[60,200]$ and 10 realizations.

\begin{tabular}{|c|c|c|}
\hline & PI & IKF \\
\hline$m_{e}$ & {$\left[\begin{array}{l}4.19 \times 10^{3} \\
4.64 \times 10^{2} \\
7.00 \times 10^{2} \\
1.59 \times 10^{4}\end{array}\right]$} & {$\left[\begin{array}{l}2.54 \times 10^{3} \\
4.00 \times 10^{3} \\
2.92 \times 10^{3} \\
1.58 \times 10^{4}\end{array}\right]$} \\
\hline \multirow{2}{*}{$\mathcal{V}_{e}$} & {$\left[\begin{array}{c}2.19 \times 10^{4} \\
7.91 \times 10^{2} \\
1.44 \times 10^{3} \\
7.34 \times 10^{3}\end{array}\right]$} & {$\left[\begin{array}{l}2.56 \times 10^{3} \\
2.87 \times 10^{3} \\
3.45 \times 10^{3} \\
1.00 \times 10^{4}\end{array}\right]$} \\
\hline$\Pi_{e}$ & {$\left[\begin{array}{c}2.07 \times 10^{5} \\
3.00 \times 10^{3} \\
6.40 \times 10^{3} \\
1.98 \times 10^{6}\end{array}\right]$} & {$\left[\begin{array}{c}5.86 \times 10^{4} \\
2.49 \times 10^{5} \\
7.86 \times 10^{4} \\
1.94 \times 10^{6}\end{array}\right]$} \\
\hline$\Sigma_{\Pi_{e}}$ & $2.20 \times 10^{6}$ & $2.32 \times 10^{6}$ \\
\hline
\end{tabular}

while the second row is chosen to guarantee an observable pair $(\bar{A}, \bar{H})$, for example:

$$
K^{i}=\left[\begin{array}{cccc}
3 & 6 & 4.5 & 0.6 \\
0 & 0 & 0.1 & 0
\end{array}\right]^{T} .
$$

Note that the assumption that $\mu_{\infty}$ is known may be unrealistic in practice. However, the objective of this section is simply to illustrate the theoretical result of Theorem 2. The gains $K^{x}$ and $K^{\alpha}$ are tuned using calibration by numerically solving (13). The resulting closed-loop poles are:

$$
\lambda_{P I}=\left[\begin{array}{lllll}
0.3411 \pm 0.0110 i & 0.7766 & 0.9208 & 0.9578 & 0.9899
\end{array}\right]^{T} .
$$

The IKF is tuned as in Section 5.2.1.

Figure 8 and Table 5 compare the performance of the PI observer and IKF. The two estimators perform similarly well for $x_{2}$ and $x_{3}$, but IKF is better for $x_{1}$ and $x_{4}$. The reason is that the PI observer is tuned to perform well for the output variables $x_{2}$ and $x_{3}$, while IKF performs equally well for all state variables.

A comparison of Figures 2 and 8 shows that using $\mu_{\infty}$ to design $K^{i}$ does not affect much the estimation of the measured variables but has a large effect on the unmeasured ones for both the PI observer and IKF. The design of an appropriate $K^{i}$, which attempts to both satisfy $\mu_{\infty}=K^{i} c_{i}$ and guarantee the observability of $(\bar{A}, \bar{H})$, represents a topic for further research.

\section{Conclusions}

This paper has investigated the design of observers with the objective of estimating accurately a subset of the states. It is shown that it is possible to eliminate the steady-state offset in the variables 

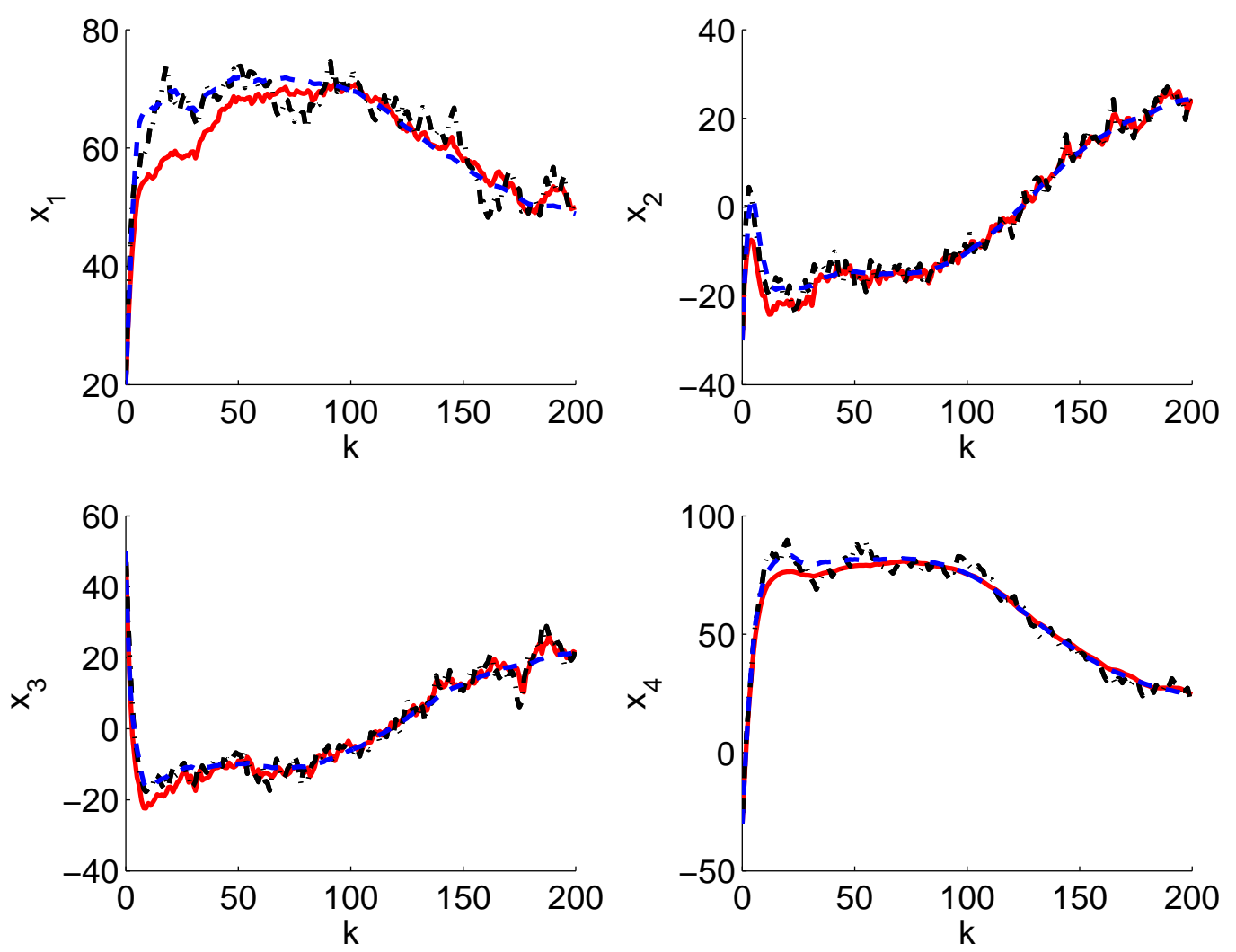

Figure 8: Performance of the PI observer and IKF for one realization in Simulation 1 (constant disturbances; PI observer tuned to eliminate bias in all states). Plant - (black) dash-dotted line; PI observer - (red) solid line; IKF - (blue) dashed line.

Table 5: Performance of the PI observers and IKF in Simulation 1 (constant disturbances; PI observer tuned to eliminate bias in all states) for $k \in[60,200]$ and 10 realizations.

\begin{tabular}{|c|c|c|}
\hline & PI observer & IKF \\
\hline$m_{e}$ & {$\left[\begin{array}{c}1.64 \times 10^{2} \\
6.89 \times 10^{1} \\
9.21 \times 10^{1} \\
1.19 \cdot 10^{2}\end{array}\right]$} & {$\left[\begin{array}{c}1.05 \times 10^{2} \\
7.92 \times 10^{1} \\
1.31 \times 10^{2} \\
7.77 \times 10^{1}\end{array}\right]$} \\
\hline \multirow{2}{*}{$\mathcal{V}_{e}$} & {$\left[\begin{array}{c}2.98 \times 10^{3} \\
5.65 \times 10^{2} \\
9.33 \times 10^{2} \\
2.10 \times 10^{3}\end{array}\right]$} & {$\left[\begin{array}{c}2.48 \times 10^{3} \\
8.99 \times 10^{2} \\
1.40 \times 10^{3} \\
1.91 \times 10^{3}\end{array}\right]$} \\
\hline$\Pi_{e}$ & {$\left[\begin{array}{c}3.28 \times 10^{3} \\
6.18 \times 10^{2} \\
1.03 \times 10^{3} \\
2.27 \times 10^{3}\end{array}\right]$} & {$\left[\begin{array}{c}2.61 \times 10^{3} \\
9.69 \times 10^{2} \\
1.57 \times 10^{3} \\
1.98 \times 10^{3}\end{array}\right]$} \\
\hline$\Sigma_{\Pi_{e}}$ & $7.21 \times 10^{3}$ & $7.14 \times 10^{3}$ \\
\hline
\end{tabular}


of interest by appropriately tuning a $\mathrm{P}$ observer based on the knowledge of the asymptotic value of the process noise. However, on-line retuning would be required when the disturbances change.

To circumvent this problem, integral observers do include auxiliary states that can absorb the effect of unknown disturbances and remove it from the output estimates. This work has shown that the observer poles can be placed arbitrarily even for the case where not all the states are measured. This leads to a stable observer and asymptotically unbiased output estimates. In fact, the simplest observer structure that guarantees both arbitrary pole placement and unbiased output estimation has been identified. Note also that unbiased estimation of all the states is possible if the gain matrix $K^{i}$ spans the disturbance space.

The choice of $K^{i}$ in the null space of the matrix $M_{1}$ guarantees the observability of $(\bar{A}, \bar{H})$ and thus observer stability and unbiased output estimation. However, $K^{i}$ in the null space of $M_{1}$ is only a sufficient condition. This means that one can very well have a $K^{i}$ that is not in the null space of $M_{1}$ but still guarantees observability. Hence, there exists a certain flexibility in the choice of $K^{i}$, which would guarantee unbiased estimation of all the state variables upon knowledge of $\mu_{\infty}$.

It has also been shown that observer tuning can be carried out using experimental data and numerical optimization using a calibration-like approach. The PI observer tuned this way compared favorably with IKF, which in this work used perfect knowledge of the process and measurement noise covariances.

The analysis part of the paper is limited to LTI systems in the presence of asymptotically constant disturbances. Both piecewise-constant and time-varying disturbances have been considered. As a general rule, integral observers can cope with slowly-varying disturbances, provided the observer dynamics are fast compared to the disturbance changes. However, being able to handle faster timevarying disturbances would most likely require the use of high-gain observers as proposed in [25], which however is rather impractical in the presence of measurement noise and process uncertainties.

\section{References}

[1] A. Alessandri, M. Baglietto, and G. Battistelli. Robust receding-horizon state estimation for uncertain discrete-time linear systems. Systems and Control Letters, 54:627-643, 2005.

[2] D. Andrisani and C. F. Gau. Estimation using a multirate filter. IEEE Trans. Autom. Contr., $32(7): 653-656,1987$.

[3] O. Y. Bas, B. Shafai, and S. P. Linder. Design of optimal gains for the proportional integral Kalman filter with application to single particle tracking. In Proceedings of the IEEE Conference on Decision and Control, pages 4567-4571, Phoenix, Arizona, USA, 1999.

[4] O. Bernard and J. L. Gouze. Closed-loop observers bundle for uncertain biotechnological models. J. Process Contr., 14:765-774, 2004.

[5] L. Bodizs. Preferential Estimation. PhD thesis, No. 4004, École Polytechnique Fédérale de Lausanne, Switzerland, 2008. 
[6] E. Bullinger and F. Allgöwer. An adaptive high-gain observer for nonlinear systems. In Proceedings of the IEEE Conference on Decision and Control, pages 4348-4353, San Diego, California, USA, 1997.

[7] C. T. Chen. Linear System Theory and Design. Oxford University Press, 1999.

[8] J. Chen, R. J. Patton, and H. Y. Zhang. Design of unknown input observers and robust fault detection filters. Int. J. Contr., 63(1):85-105, 1996.

[9] M. Darouach and M. Zasadzinski. Unbiased mimimum variance estimation for systems with exogenous inputs. Automatica, 33(4):717-719, 1997.

[10] Ph. de Vallière and D. Bonvin. Application of estimation techniques to batch reactors - II. Experimental studies in state and parameter estimation. Comp. Chem. Eng., 13:11-20, 1989.

[11] D. Dochain. State and parameter estimation in chemical and biochemical processes: A tutorial. J. Process Contr., 13:801-818, 2003.

[12] N. M. Faber. A closer look at the bias-variance trade-off in multivariate calibration. J. of Chemometrics, 13:185-192, 1999.

[13] B. Friedland. Treatment of bias in recursive filtering. IEEE Trans. Autom. Contr., 14:359-367, 1969.

[14] A. M. Gibon-Fargeot, H. Hammouri, and F. Celle. Nonlinear observers for chemical reactors. Chem. Eng. Sci., 49(14):2287-2300, 1994.

[15] S. Gillijns and B. De Moor. Unbiased minimum-variance input and state estimation for linear discrete-time systems. Automatica, 43(1):111-116, 2007.

[16] M. S. Grewal and A. P. Andrews. Kalman Filtering. Theory and Practice. Prentice Hall, 1993.

[17] G. Hostetter and J. S. Meditch. Observing systems with unmeasurable inputs. IEEE Trans. Autom. Contr., 18:307-308, 1973.

[18] M. Hou and R. J. Patton. Input observability and input reconstruction. Automatica, 34(6):789$794,1998$.

[19] P. K. Kitanidis. Unbiased minimum-variance linear state estimation. Automatica, 23(6):775$778,1987$.

[20] D. G. Luenberger. An introduction to observers. IEEE Trans. Autom. Contr., 16:596-602, 1971.

[21] H. Martens and T. Naes. Multivariate Calibration. John Wiley, 1989.

[22] H. H. Niemann, J. Stoustrup, B. Shafai, and S. Beale. LTR design of proportional-integral observers. Int. J. Robust Nonlinear Contr., 5:671-693, 1995.

[23] M. Pottmann, B. A. Ogunnaike, and J. S. Schwaber. Development and implementation of a high-performance sensor system for an industrial polymer reactor. Ind. Eng. Chem. Res., 44:2606-2620, 2005.

[24] H. W. Smith and E. J. Davison. Design of industrial regulators: Integral feedback and feedforward control. Proc. IEE, 119:1210-1216, 1972. 
[25] D. Soeffker, T. Yu, and P. C. Mueller. State estimation of dynamical systems with nonlinearities by using proportional-integral observers. Int. J. Systems Sci., 26:1571-1582, 1995.

[26] M. Soroush. State and parameter estimations and their applications in process control. Comp. Chem. Eng., 23:229-245, 1998.

[27] S. Tatiraju, M. Soroush, and B. A. Ogunnaike. Multirate nonlinear state estimation with application to a polymerization reactor. AIChE J., 45:769-780, 1999.

[28] N. Zambare, M. Soroush, and B. A. Ogunnaike. A method of robust multirate state estimation. J. Process Contr., 13:337-355, 2003. 